\title{
Effect of Substrate Thickness on Ejection of Phenylalanine Molecules Adsorbed on Free-standing Graphene Bombarded by $10 \mathrm{keV} \mathrm{C}_{60}$
}

\author{
M. Golunski ${ }^{\mathrm{a}}$, S.V. Verkhoturov ${ }^{\mathrm{b}}$, D.S. Verkhoturov ${ }^{\mathrm{b}}$, E.A. Schweikert ${ }^{\mathrm{b}}$, Z. Postawa $^{\mathrm{a},{ }^{*},}$
}

Molecular dynamics computer simulations have been employed to investigate the effect of substrate thickness on the ejection mechanism of phenylalanine molecules deposited on freestanding graphene. The system is bombarded from the graphene side by $10 \mathrm{keV} \mathrm{C}_{60}$ projectiles at normal incidence and the ejected particles are collected both in transmission and reflection directions. It has been found that the ejection mechanism depends on the substrate thickness. At thin substrates mostly organic fragments are ejected by direct collisions between projectile atoms and adsorbed molecules. At thicker substrates interaction between deforming topmost graphene sheet and adsorbed molecules becomes more important. As this process is gentle and directionally correlated, it leads predominantly to ejection of intact molecules. The implications of the results to a novel analytical approach in Secondary Ion Mass Spectrometry based on ultrathin free-standing graphene substrates and a transmission geometry are discussed.

Keywords: Computer simulations, sputtering, cluster projectiles, graphene, organic overlayers. 


\section{Introduction}

In recent years, cluster ion beams have attracted increasing experimental and theoretical attention due to their capacity to enhance ejection of large intact organic molecules in Secondary Ion Mass Spectrometry (SIMS) [1, 2]. One of the most successful clusters used in organic SIMS is $\mathrm{C}_{60}$ fullerene [3]. In a typical SIMS geometry the detector is located on the same side of the target as the ion gun. Usually metal or semiconductor supports are used to deposit the investigated material. A novel SIMS configuration, using transmission orientation, has been proposed recently $[4,5]$. In this orientation, the analysed organic material is deposited on one side of the ultrathin substrate, while another side is bombarded by cluster projectiles. It is argued that such geometry can be particularly attractive for analysis of small amounts of organic material, molecular nano-objects and supramolecular assemblies [5].

There are several simulations performed on $\mathrm{C}_{60}$ bombardment of graphene and graphite [4, 6-13]. However, most of these studies concentrate on defect creation in the bombarded system rather than on material ejection. Theoretical studies of sputtering of graphite by $\mathrm{keV} \mathrm{C}_{60}$ projectiles show that the sputtering yield is low $[11,13]$. Krantzman et al. have attributed this fact to a low atomic density of graphite [11], while the effect of the layered structure of graphite was emphasised by Tian et al. [13]. It also has been shown that the membrane-like structure of graphite can be made to vibrate as a result of a cluster impact $[4,8,9]$. The mesoscopic motion of created circular acoustic waves can stimulate ejection of small weakly bound molecules [8,9]. Although this mechanism may not be efficient for uplifting heavier molecules as it may not provide enough energy, it has been postulated that vibrational energy can be utilized to stimulate ionization [4]. Computer simulations of bombardment of organic molecules deposited on metal substrates show that intact molecules are emitted by low-energy collisions with ejecting substrate 
or projectile atoms [14], and/or by surface deformations occurring during crater formation [15]. As sputtering of graphite is different from sputtering of metals $[13,16]$ we would like to check if similar phenomena are present for the ultrathin graphite. Furthermore, the only theoretical study performed so far with $\mathrm{C}_{60}$ impacts in transmission geometry was done on a system of a constant thickness [4]. The goal of this paper is, therefore, to investigate processes that lead to ejection of organic molecules deposited on ultrathin free-standing graphene of various thickness bombarded by $10 \mathrm{keV} \mathrm{C}_{60}$ projectiles in a transmission orientation.

\section{Computer model}

A detailed description of the molecular dynamics computer simulations used to model cluster bombardment can be found elsewhere [1]. Briefly, the motion of particles is determined by integrating Hamilton's equations of motion. The forces among atoms in the system are described by a Reax-FF force field [17] splined at short distances with a ZBL potential to properly describe high energy collisions. The shape and size of the samples are chosen based on a visual observation of energy transfer pathways stimulated by impact of $\mathrm{C}_{60}$ projectiles. As a

result, cylindrical samples with a diameter of $400 \AA$ are used. Substrates with a thickness ranging from 2 to 16 graphene layers with a $\mathrm{HOPG}$ structure are bombarded by $10 \mathrm{keV} \mathrm{C}_{60}$ projectiles that are directed at the bottom of the sample. Ten phenylalanine molecules are deposited on the top of the graphene substrate, as shown in Figure 1. Molecules are placed away from each other to mimic submonolayer coverage. They are also located at different distance from the impact zone to probe the influence of this parameter on the mechanism of ejection. Phenylalanine molecules are selected as they are important amino acids, they are simple, yet consist of most elements that are present in biomaterials. Particles ejected both in direction of the primary beam (transmission direction) and in the opposite direction (reflection direction) are collected. Rigid 
and stochastic regions are used to simulate the thermal bath and to prevent reflection of pressure waves from the boundaries of the system $[1,18]$. The simulations are run at $0 \mathrm{~K}$ target temperature in an NVE ensemble and extend up to $10 \mathrm{ps,} \mathrm{which} \mathrm{is} \mathrm{long} \mathrm{enough} \mathrm{to} \mathrm{achieve}$ saturation in the ejection yield vs time dependence. Eight impact points within the linear impact zone represented by white line in Figure $\mathbf{1}$ are chosen to achieve statistically more reliable data.

\section{Results and discussion}

Numbers of particles ejected from systems of various thickness by $10 \mathrm{keV} \mathrm{C}_{60}$ impacts are given in Table 1. While it is evident that the yields depend on substrate thickness, the actual dependence is different for different particles. The number of projectile atoms penetrating the sample decreases with a thickness of the substrate. Interestingly, almost no projectile atoms are backscattered even from the thickest system, which means that non-ejected atoms are implanted into the sample. The ejection yield of substrate atoms in the transmission direction depends nonmonotonically on the substrate thickness. At first, the signal increases as more carbon atoms become available for ejection when the sample is getting thicker. However, with the increase of the substrate thickness more primary kinetic energy is sacrificed to penetrate through a thicker solid. As a result, less energy is available near the surface from where the ejection occurs, and, ultimately, the signal decreases. Atoms originally located in all layers are recorded in the ejected flux, although ejection from the topmost layer is dominant. A similar dependence on the substrate thickness occurs for atoms originating from phenylalanine molecules, however, the reason of such behavior has to be different than for substrate atoms as the number of molecules available for ejection is constant. For substrates composed of up to 6 graphene layers predominantly molecular fragments are ejected. For thick substrates $(\geq 12 \mathrm{~L})$ the ejected flux is composed entirely from intact molecules. 
Cross sectional views of the temporal evolution of 2,8 and 16-layer systems are shown in Figure 2 to identify processes responsible for molecular ejection. In all systems $\mathrm{C}_{60}$ projectiles decompose into smaller pieces almost immediately after the impact. As indicated in Table $\mathbf{1}$ at the 2-layer (2L) sample almost all projectile atoms penetrate through the substrate. Nevertheless, even in this system a projectile-graphene interaction is surprisingly strong, as already a half of the primary kinetic energy is transferred to the substrate. Most of this energy is carried away by ejected substrate particles. Ejection of both projectile and substrate atoms is forward directed. Ejecting atoms can collide with organic molecules causing their ejection. However, the average kinetic energy of ejected projectile and substrate atoms is high. As a result, such collisions lead to molecular fragmentation, as seen for molecule B in Figure 2a. The projectile impact also leads to a creation of cylindrical acoustic waves that propagate in the graphene outward from the point of impact with a maximum amplitude of 1 Å. It was reported that these waves are capable to uplift benzene and cumene molecules $[8,9]$. However, no similar phenomenon has been observed in our study.

A dramatic alteration of a substrate structure caused by $\mathrm{C}_{60}$ impact is observed at thicker systems, as shown in Figure $\mathbf{2 b}$ for the $8 \mathrm{~L}$ graphene. The projectile is more efficiently decelerated, depositing almost all of its primary kinetic energy into the substrate. Part of this energy is used to eject substrate atoms in the forward direction. The remaining part is used to deform the substrate. Soon after the projectile impact substrate integrity is compromised. Near the point of impact graphene sheets become separated from each other and bend up in a direction parallel to the movement of incoming projectile. Finally, a cylindrical opening is formed surrounded by elevated rim at the top surface of the sample. 
While the average kinetic energy of ejected projectile and substrate atoms is smaller than in the $2 \mathrm{~L}$ system, it is still high enough to fragment molecule B. However, the unfolding of graphene sheets, which works like a catapult can also eject other molecules, as visible for molecule C. As the process is gentle and occurs in a coordinated fashion, the ejected molecules are not fragmented. This process supplements molecular ejection by collisions leading to an increase of the organic signal and to the appearance of intact molecules in the sputtered flux. A similar mechanism was observed during crater formation at the metal surfaces bombarded by cluster projectiles [15]. However, surface deformation observed in graphene extends to a much larger lateral distance making this process much more efficient than in metals. It is also worth mentioning that catapult-like sheets movement is almost absent at the surface directly hit by a projectile. This observation indicates that a transmission geometry is a better choice for efficient ejection of adsorbed molecules, at least, for ultra-small coverages.

The process of graphene unfolding becomes less efficient with a further increase of a substrate thickness. As shown in Figure 2c, the 16L substrate is too thick to be perforated by a $10 \mathrm{keV} \mathrm{C}_{60}$ projectile. In this case no unfolding is present. It is interesting to note that all projectile atoms become implanted inside the substrate. Such behavior is different than observed during $\mathrm{C}_{60}$ bombardment of metals, where almost all projectile atoms are backscattered into the vacuum $[16,18]$. There is also a big difference in sputtering yields recorded from these two materials. The sputtering yield is large for metals and low for graphite. For instance, a sputtering yield of $41 \pm 3$ was reported for $20 \mathrm{keV} \mathrm{C}_{60}$ impact on graphite [13], while ejection of almost 500 atoms was observed from $\operatorname{Ag}(111)$ at the same impact conditions [16]. Barely 9 carbon atoms are sputtered on average, i.e. ejected in reflection direction, from our thickest system bombarded by $10 \mathrm{keV} \mathrm{C}_{60}$. The different behavior of implantation and sputtering processes is a consequence of 
a different redistribution of the deposited energy in these two systems. In metals, $\mathrm{C}_{60}$ is quickly decelerated depositing its kinetic energy close to the bombarded surface $[1,16]$. The density of deposited energy is high and the energy is redistributed initially by spherical pressure pulses. Atoms are relocated and a crater is formed. Substrate atoms are ejected from the corona of the crater by a fluid flow motion, which is supplemented at later time by atom effusion from the inside of a formed crater $[15,16,19]$. Most of impinging projectile atoms either immediately rebound into the vacuum when colliding with heavier Ag surface atoms, or are implanted inside the volume, where the crater will be formed, and will be ejected during this process.

The behavior of graphite is different. Firstly, graphite has a low number density which leads to a lower density of deposited energy. As a result, both the number of carbon atoms taking place in the flow and the strength of the outward pressure pulse is reduced [11, 19]. However, the most critical factor for sputtering is the layered structure of graphite [13]. Computer simulations show that the energy transfer is more efficient along the graphene sheets than in the vertical direction $[9,13]$. Consequently, energy that normally would be directed towards the surface and contribute to particle ejection, is now laterally channeled away. Finally, the binding between carbon atoms in graphite is stronger than binding between $\mathrm{C}$ and $\mathrm{Ag}$ atoms. A stronger binding combined with a lower efficiency of vertical movement is responsible for the absence of backreflection of projectile atoms in graphite.

Although projectile atoms are not penetrating through the substrate, the upper surface of the 16L system bulges outward during projectile deceleration as shown in Figure 2c. This motion is energetic enough to stimulate ejection of physisorbed phenylalanine molecules, as shown at the bottom panel. The efficiency of this process depends on the substrate thickness. One may expect it to be the most efficient for substrates where the largest fraction of the primary 
kinetic energy is delivered to the largest area of the topmost graphene sheet. As the bulging process is gentle and no energetic projectile or substrate atoms are ejected, only intact molecules are emitted. The simulations presented here are done for a single projectile kinetic energy. It would be interesting to verify how the primary kinetic energy influences ejection processes. For instance, is there a scaling effect of energy versus number of layers for the cases shown in Figure 2? This topic is currently investigated and will be published elsewhere.

\section{Conclusions}

Molecular dynamics computer simulations have been performed to study the effect of the sample thickness on the ejection mechanism of phenylalanine molecules deposited on free-standing graphene bombarded by $10 \mathrm{keV} \mathrm{C}_{60}$. The mechanism of molecular ejection depends on the substrate thickness. For thin substrates, which are perforated by projectile atoms, collisions between projectile/substrate atoms and adsorbed molecules is the main mechanism of molecular ejection. At thicker substrates the catapult-like action of the unfolding graphene sheets becomes important. The efficiency of this process depends on the substrate thickness reaching the maximum for 8-layer system, at least, for $10 \mathrm{keV} \mathrm{C}_{60}$ projectiles. When the graphite layer becomes too thick to be perforated, the ejection of adsorbed molecules occurs due to a bulging out of the surface. Although acoustic waves are created by the $\mathrm{C}_{60}$ impact, interaction with these waves does not lead to ejection of phenylalanine molecules.

Based on our results a few comments can be made about applicability of ultrathin graphite substrates for SIMS. Before doing this it should be pointed out, however, that the data presented in this paper relate to the sputtering of neutral organic molecules while ions are recorded in SIMS. To simulate ion emission, ionization and neutralization processes should be included into the model calculations, which is still an unresolved problem. One should refrain, 
therefore, from quantitative comparisons between emission enhancements of ions and neutrals. However, analysis of the structural modifications of the bombarded system and its influence on particle emission is applicable to the study of both ions and neutrals. Our study confirms that graphene supports have several advantages, as compared to traditional metal or semiconductor substrates especially for analysis of sub-monolayer amounts of analyte. Firstly, the extremely small thickness of the support results in a small amount of emitted substrate material especially for $2 \mathrm{~L}$ or $4 \mathrm{~L}$ systems. As a result, there is a minimal interference between substrate and analyzed signal. Furthermore, the emission processes favor ejection of molecules towards the detector improving detection efficiency.

Finally, it also should be stressed that our findings are valid for sub-monolayer coverages. For thicker organic overlayers, a significant portion of the primary kinetic energy will be directly deposited inside the organic layer. The propagation of this energy within the overlayer will lead to molecular ejection and, most probably, will dominate ejection process. However, this phenomenon will be examined in future work.

\section{Acknowledgments}

The authors gratefully acknowledge financial support from the Polish National Science Center, Program No. 2013/09/B/ST4/00094, 2015/19/B/ST4/01892 and from the US National Science Foundation grant CHE-1308312.

\section{References}

[1] B.J. Garrison, Z. Postawa, Mass Spectrometry Reviews, 27 (2008) 289-315 and references therein. 
[2] N. Winograd, Analytical Chemistry, 77 (2005) 142A-149A and references therein.

[3] D. Weibel, S. Wong, N. Lockyer, P. Blenkinsopp, R. Hill, J.C. Vickerman, Analytical Chemistry, 75 (2003) 1754-1764.

[4] S.V. Verkhoturov, S. Geng, B. Czerwinski, A.E. Young, A. Delcorte, E.A. Schweikert, J. Chem. Phys., 143 (2015) 164302-1.

[5] M.J. Eller, C.K. Liang, S. Della-Negra, A.B. Clubb, H. Kim, A.E. Young, E.A. Schweikert, J. Chem. Phys., 142 (2015) 044308.

[6] R. Smith, R.P. Webb, Proceedings of the Royal Society of London Series a-Mathematical Physical and Engineering Sciences, 441 (1993) 495-499.

[7] M. Kerford, R.P. Webb, Nuclear Instruments and Methods in Physics Research Section B: Beam Interactions with Materials and Atoms, 153 (1999) 270-274.

[8] M. Kerford, R.P. Webb, Nuclear Instruments and Methods in Physics Research Section B: Beam Interactions with Materials and Atoms, 180 (2001) 44-52.

[9] R.P. Webb, Radiat. Eff. Defects Solids, 162 (2007) 567-572.

[10] C. Anders, H. Kirihata, Y. Yamaguchi, H.M. Urbassek, Nuclear Instruments \& Methods in Physics Research Section B-Beam Interactions with Materials and Atoms, 255 (2007) 247-252.

[11] K.D. Krantzman, R.P. Webb, B.J. Garrison, Applied Surface Science, 255 (2008) 837-840.

[12] S.J. Zhao, J.M. Xue, L. Liang, Y.G. Wang, S. Yan, Journal of Physical Chemistry C, 116 (2012) 11776-11782.

[13] J.T. Tian, T. Zheng, J.Y. Yang, S.Y. Kong, J.M. Xue, Y.G. Wang, K. Nordlund, Applied Surface Science, 337 (2015) 6-11.

[14] B.J. Garrison, A. Delcorte, K.D. Krantzman, Accounts Chem. Res., 33 (2000) 69-77.

[15] Z. Postawa, Applied Surface Science, 231 (2004) 22-28. 
[16] Z. Postawa, B. Czerwinski, M. Szewczyk, E.J. Smiley, N. Winograd, B.J. Garrison, Journal of Physical Chemistry B, 108 (2004) 7831-7838.

[17] L.C. Liu, Y. Liu, S.V. Zybin, H. Sun, W.A. Goddard, J. Phys. Chem. A, 115 (2011) 1101611022.

[18] Z. Postawa, B. Czerwinski, M. Szewczyk, E.J. Smiley, N. Winograd, B.J. Garrison, Analytical Chemistry, 75 (2003) 4402-4407.

[19] M.F. Russo, B.J. Garrison, Analytical Chemistry, 78 (2006) 7206-7210. 


\section{Table captions}

Table 1. The total number of atoms ejected in transmission (Ejected) and reflection (Sputtered/Reflected) directions, and the average kinetic energy $\mathrm{KE}_{\mathrm{ave}}$ of atoms ejected from a free-standing graphene of various thickness bombarded by $10 \mathrm{keV}$ $\mathrm{C}_{60}$ projectiles. The term "organic atoms" relates to atoms of phenylalanine molecules. The numbers in round brackets depict relative contribution of intact phenylalanine molecules in the ejected flux.

\begin{tabular}{|c|c|c|c|c|c|c|c|}
\hline \multirow{2}{*}{$\begin{array}{l}\text { Number } \\
\text { of } \\
\text { substrate } \\
\text { layers }\end{array}$} & \multicolumn{3}{|c|}{ Projectile atoms } & \multicolumn{3}{c|}{ Substrate atoms } & \multicolumn{2}{|c|}{$\begin{array}{l}\text { "Organic } \\
\text { atoms" }\end{array}$} \\
\cline { 2 - 8 } & Ejected & Reflected & $\begin{array}{l}\mathrm{KE}_{\text {ava }} \\
{[\mathrm{eV}]}\end{array}$ & Ejected & Sputtered & $\begin{array}{c}\mathrm{KE}_{\text {ava }} \\
{[\mathrm{eV}]}\end{array}$ & Ejected \\
\hline 2 & $57 \pm 1$ & 0.0 & 89 & $49 \pm 2$ & $0.13 \pm 0.1$ & 59 & $21 \pm 4 \quad(0 \%)$ \\
\hline 4 & $46 \pm 1$ & 0.2 & 44 & $155 \pm 4$ & $1.1 \pm 0.5$ & 24 & $39 \pm 5(7 \%)$ \\
\hline 6 & $32 \pm 2$ & 0.2 & 16 & $259 \pm 5$ & $0.9 \pm 0.6$ & 10 & $49 \pm 4(23 \%)$ \\
\hline 8 & $12 \pm 1$ & 0.3 & 13 & $205 \pm 13$ & $3.3 \pm 1.0$ & 3 & $60 \pm 4(62 \%)$ \\
\hline 12 & $0.2 \pm 0.2$ & 0.5 & 5 & $0.3 \pm 0.2$ & $5 \pm 2$ & 2 & $81 \pm 5(100 \%)$ \\
\hline 16 & 0.0 & 1.2 & 0 & 0.0 & $9 \pm 3$ & 0 & $64 \pm 5(100 \%)$ \\
\hline
\end{tabular}




\section{Figure captions}

Figure 1. The model system used to study ejection processes of phenylalanine molecules deposited on free-standing graphene. Numbers indicate distance from the centre of the system. The white line depicts impact points.

Figure 2. Cross-sectional view of the temporal evolution of a typical collision event leading to ejection of atoms due to $10 \mathrm{keV} \mathrm{C}_{60}$ bombardment of a system composed from phenylalanine molecules deposited on a) 2, b) 8 and c) 16 graphene layers. A slice $15 \AA$ wide of the system centred at the impact point is shown. The dashed lines in the background are separated by $10 \AA$. 


\section{$88 \mathrm{~A}=66 \mathrm{~A}$

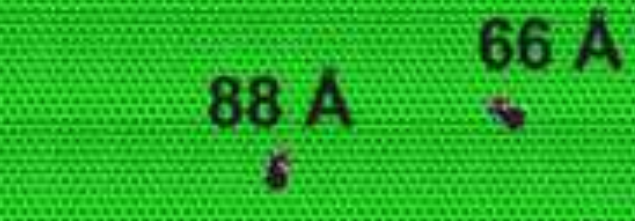

$(C)^{\circ}, 44 \mathrm{~A}$

$110 \mathrm{~A}$

?

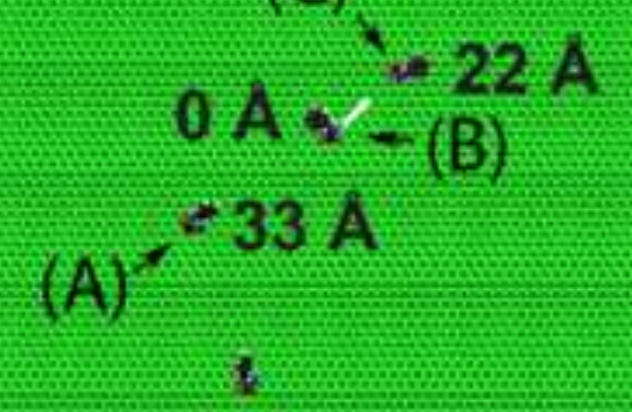

$(A)^{\prime}=33 A^{*}$

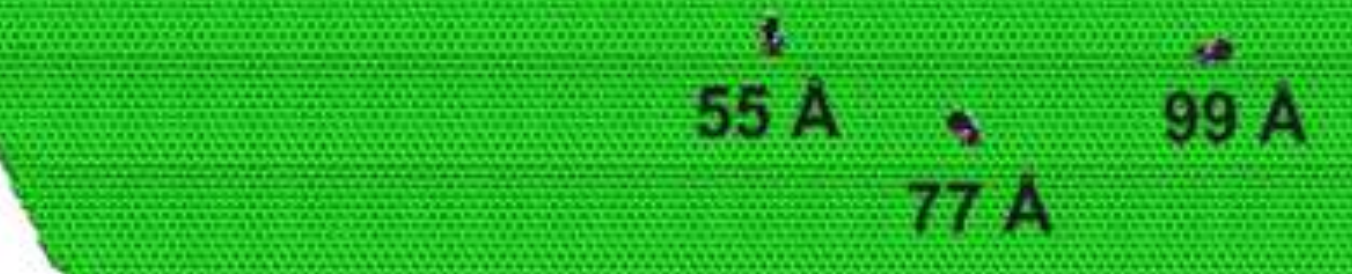


a) $\quad b$

$100 \mathrm{fs}$ b)

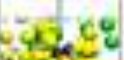

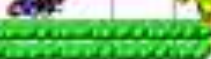

\section{$\uparrow$}

$600 \mathrm{fs}$

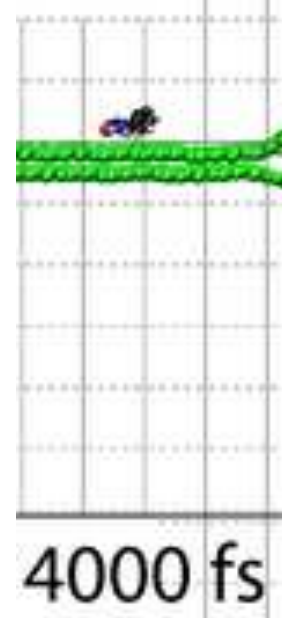

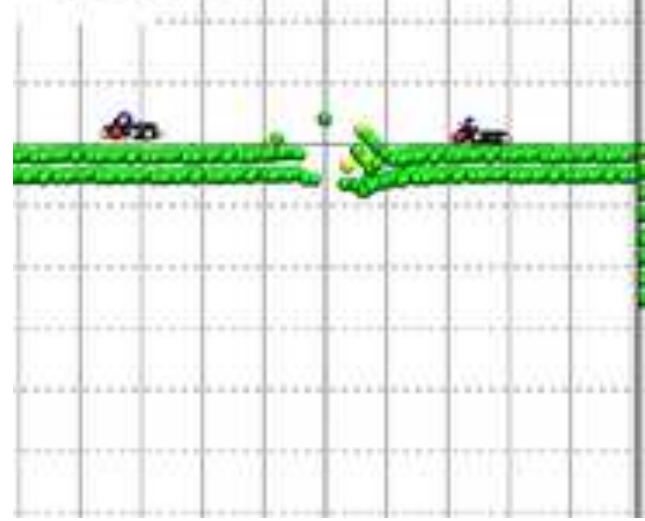
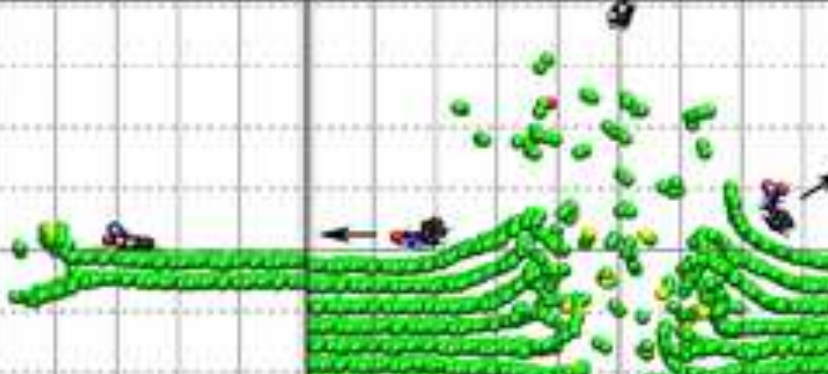

C)

(A) (B) (C)
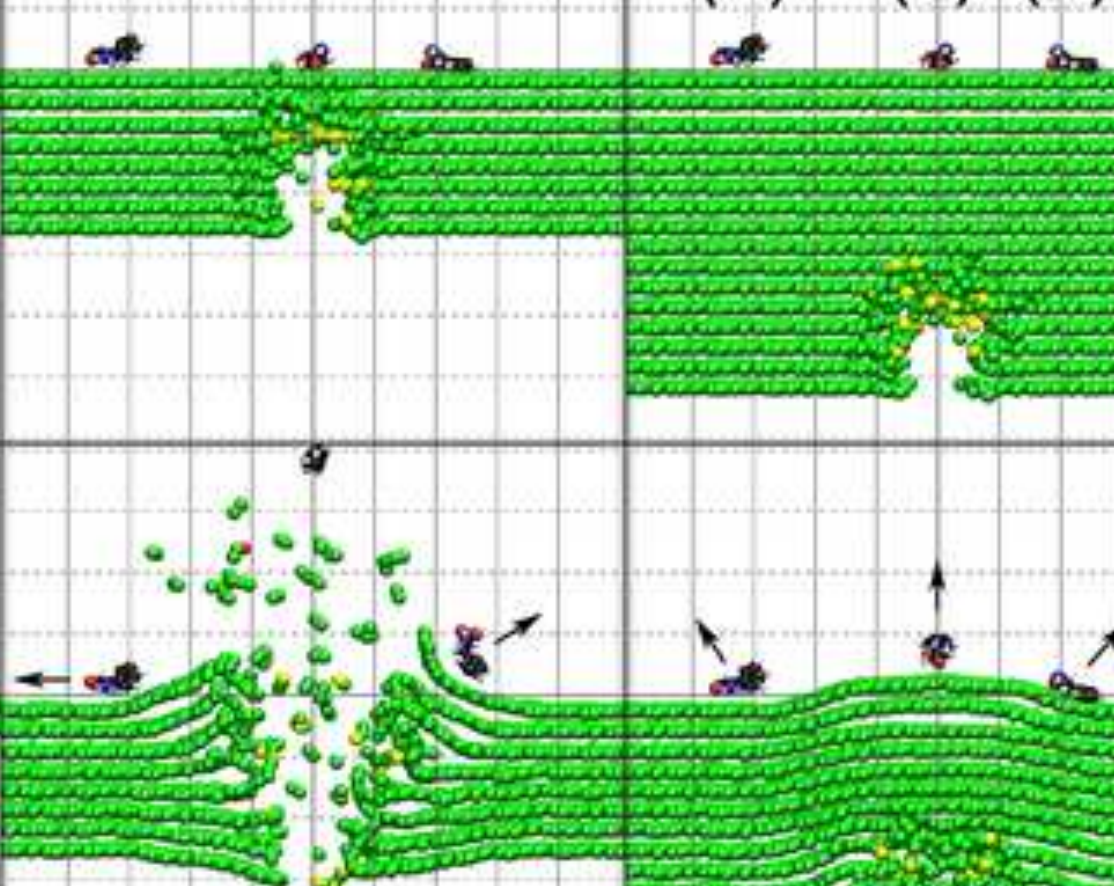

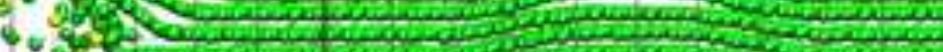
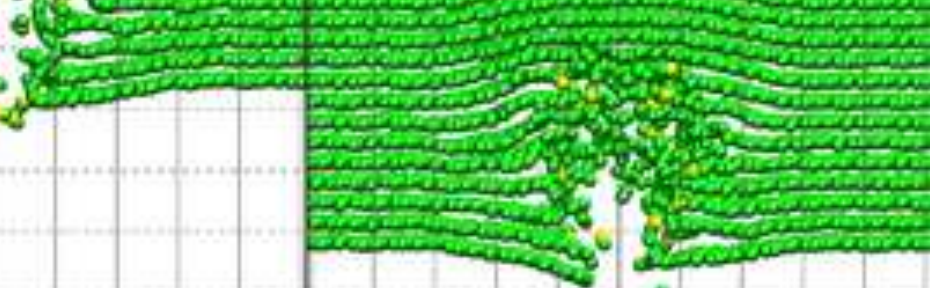

$4000 \mathrm{fs}$

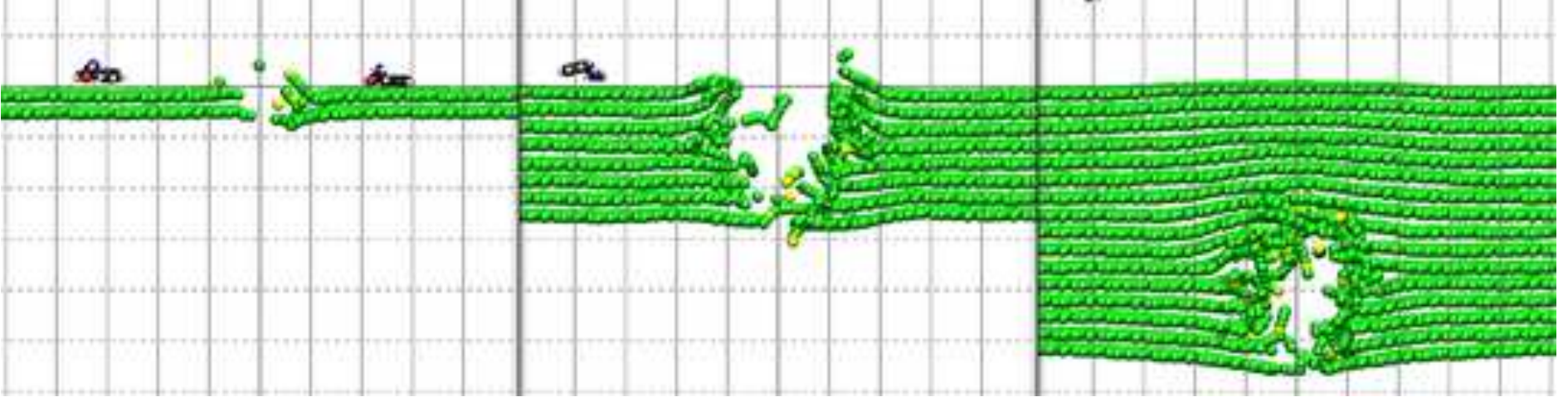

